\title{
Making sense in humans
}

In human peripheral blood there are two main B cell populations: a naive $\operatorname{IgM}^{+} \mathrm{CD} 27^{-}$population that has unmutated immunoglobulin genes and a class-switched memory $\mathrm{IgG}^{+} \mathrm{CD} 27^{+}$population. $\operatorname{IgM}^{+} \mathrm{CD} 27^{+}$ memory B cells have also been identified, but the generation and immunological function of these cells are poorly understood. Now, Ralf Küppers and colleagues report that human $\operatorname{IgM}^{+}$memory B cells are more similar to $\mathrm{IgG}^{+}$memory B cells than to naive $\mathrm{B}$ cells. Human $\operatorname{IgM}^{+}$ memory B cells can re-enter germinal centre (GC) reactions upon antigen re-encounter and they can interact with neutrophils in early inflammatory responses in vitro.

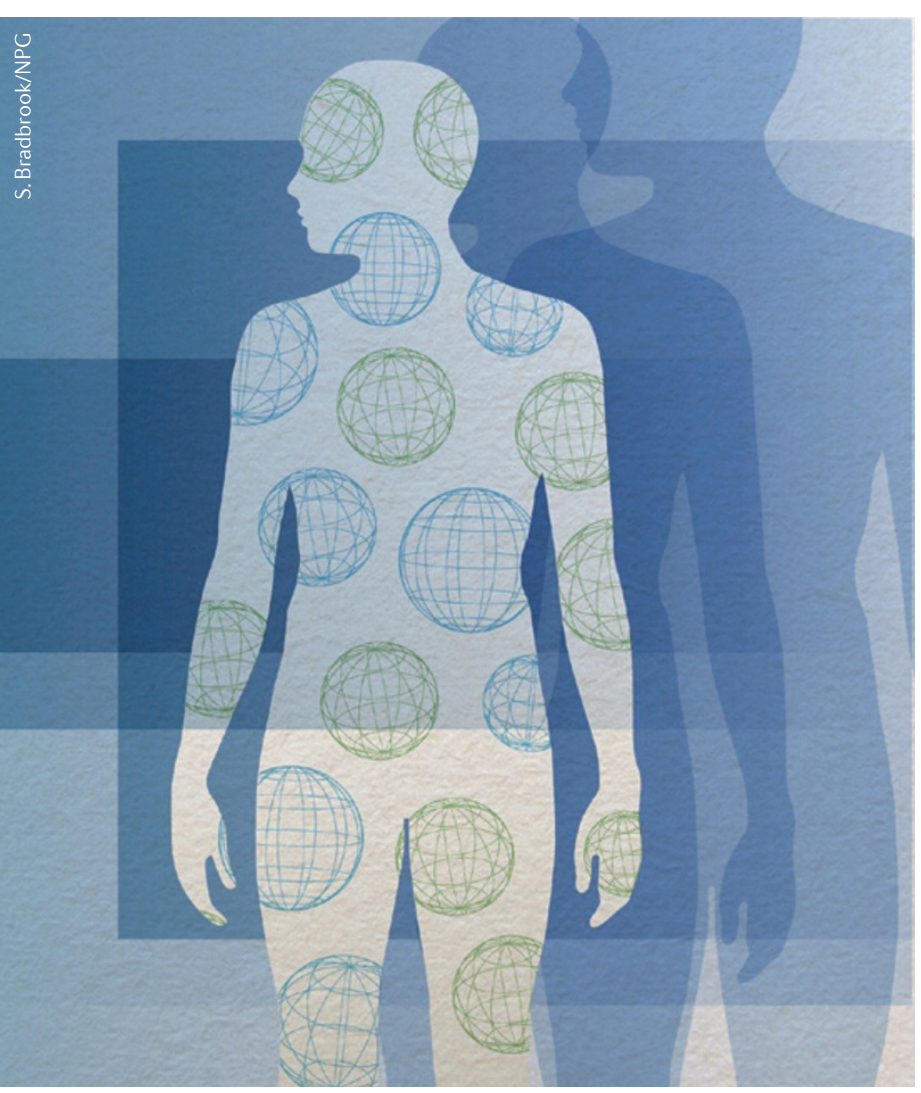

$\operatorname{IgM}^{+}$memory

$B$ cells have chemotactic activity

5

To characterize different human B cell subsets, the authors compared the transcriptional expression profiles of $\mathrm{IgM}^{+} \mathrm{CD} 27^{-}, \mathrm{IgG}^{+} \mathrm{CD} 27^{+}$and $\mathrm{IgM}^{+} \mathrm{CD} 27^{+} \mathrm{B}$ cells. The two $\mathrm{CD} 27^{+}$ memory B cell subsets showed substantial similarities and were clearly different from the naive CD27$B$ cells. Gene set enrichment analysis identified shared gene signatures that were associated with a memory B cell phenotype, including genes associated with enhanced antigen responsiveness and plasmablast differentiation.

Next, the authors investigated differences between $\operatorname{IgG}^{+}$and $\operatorname{IgM}^{+}$ memory B cells; pairwise gene comparisons revealed more than 400 genes with at least a twofold difference in expression between the two B cell subsets. They identified genes encoding key molecules that have specific functions in $\operatorname{IgM}^{+}$memory B cells, including carcinoembryonic antigenrelated cell adhesion molecule 1 (CEACAM1) and CC-chemokine receptor 2 (CCR2). In vitro chemotactic assays with soluble CEACAM8 (sCEACAM8) and CC-chemokine ligand 2 (CCL2) showed that $\operatorname{IgM}^{+}$ memory B cells have chemotactic activity towards both ligands. Furthermore, $\operatorname{IgM}^{+}$memory B cells differentiated more readily into antibody-secreting cells than $\operatorname{IgG}^{+}$ memory B cells and naive B cells in response to sCEACAM8 stimulation. Thus, by expressing different surface receptors, $\operatorname{IgM}^{+}$memory B cells can respond to stimulation by specific signals, which can lead to increased migratory capacity and differentiation into antibody-secreting cells.

Interestingly, both sCEACAM8 and CCL 2 are known to be secreted by neutrophils in early phases of inflammatory responses and these molecules help to recruit leukocytes.
Co-cultures of neutrophils and $\operatorname{IgM}^{+}$ memory B cells showed that activated neutrophils could stimulate $\operatorname{IgM}^{+}$ memory B cells to differentiate and secrete IgM or class switch to IgG2.

Finally, the authors investigated whether human $\operatorname{IgM}^{+}$and $\operatorname{IgG}^{+}$ memory B cells show a distinct memory response to antigen recall. $\operatorname{IgM}^{+}$ memory B cells showed greater migration towards signals that direct homing to $\mathrm{B}$ cell follicles than naive and $\mathrm{IgG}^{+}$memory B cells, whereas the naive and $\mathrm{IgG}^{+} \mathrm{B}$ cell subsets preferentially migrated towards signals that direct plasma cell homing. This result suggests that $\mathrm{IgM}^{+}$memory $\mathrm{B}$ cells can re-enter GC reactions. Indeed, they found that human $\operatorname{IgM}^{+}$ memory B cells upregulated the expression of the GC-associated transcription factor B cell lymphoma 6 (BCL-6), which indicates that $\operatorname{IgM}^{+}$memory B cells preferentially adopted a pre-GC B cell phenotype. However, following T cell-independent antigen stimulation, $\operatorname{IgM}^{+}$memory B cells also differentiated into plasma cells, as they adopted plasma cell morphology and downregulated their expression of BTB and CNC homologue 2 (BACH2). By contrast, human $\mathrm{IgG}^{+}$memory B cells primarily differentiated into plasma cells following re-encounter with either T celldependent or T cell-independent antigen.

Together, these results increase our understanding of human peripheral blood $\operatorname{IgM}^{+}$memory B cells and reveal the unexpected functional plasticity of these cells.

Elisabeth Kugelberg

ORIGINAL RESEARCH PAPER Seifert, M. et al. Functional capacities of human IgM memory B cells in early inflammatory responses and secondary germinal center reactions. Proc. Natl Acad. Sci. USA http://dx.doi.org/10.1073/pnas.1416276112 (2015) 